\title{
INFLUENCE OF A SMARTPHONE USE ON DYNAMIC BALANCE IN HEALTHY ADOLESCENTS
}

original paper

() Wroclaw University of Health and Sport Sciences

DOI: https://doi.org/10.5114/hm.2021.106171

\section{MARIAN M. SHAFEEK ${ }^{1}$, HANAN HOSNY M. BATTESHA ${ }^{2}$, AMIR N. WADEE $^{3}$, HODA M. IBRAHIM ${ }^{4}$}

${ }^{1}$ Department of Physical Therapy for Pediatrics and Its Surgery, Faculty of Physical Therapy, Modern University for Technology and Information, Cairo, Egypt

${ }^{2}$ Department of Physical Therapy for Neuromuscular Disorders and Its Surgery, Faculty of Physical Therapy, Modern University for Technology and Information, Cairo, Egypt

${ }^{3}$ Department of Physical Therapy for Basic Sciences, Faculty of Physical Therapy, Cairo University, Cairo, Egypt

${ }^{4}$ Department of Physical Therapy for Musculoskeletal Disorders and Its Surgery, Faculty of Physical Therapy,

Modern University for Technology and Information, Cairo, Egypt

\begin{abstract}
Purpose. The aim of the study was to detect the immediate and late effects of using a smartphone for 30 consecutive minutes on dynamic balance in healthy adolescents.

Methods. Overall, 96 healthy adolescents of both genders, aged 15-18 years, were randomly assigned to the study and the control group. The subjects in the study group used a smartphone for 30 consecutive minutes; smartphones were not allowed in the control group. A Biodex system was used to assess the dynamic balance initially, as well as immediately after and 1 hour after the intervention.

Results. MANOVA test revealed that there were statistically significant differences in the overall stability index and anteroposterior stability index ( $p=0.002$ and 0.04 , respectively), with a statistically insignificant difference in the mediolateral stability index $(p=0.46)$ within the study group. Significant differences were observed in the immediate measurements of both overall stability index and anteroposterior stability index ( $p=0.0001$ and 0.03 , respectively), while statistically insignificant differences were noted in the measurements of mediolateral stability index between the groups.

Conclusions. The dynamic balance decreased after 30 consecutive minutes of smartphone use, so care should be taken to avoid accidents while walking or performing other daily activities. This effect, however, disappeared 1 hour later.
\end{abstract}

Key words: smartphone, dynamic balance, adolescent

\section{Introduction}

The smartphone has already become a part of adolescents' daily life; it offers many conveniences, but its negative effects should not be overlooked. Adolescents turn out to be habitually dependent on smartphones and when they do not use them, they feel nervous [1]. Smartphone usage influences clients both physically and mentally. A longer span of smartphone use causes a consistent mechanical load on the muscles and ligaments, which can result in musculoskel- etal side effects as undeniable irritation of neck and shoulders because of expanded pressure brought about by a persistently forward neck posture [2].

The increase in the using rate of a smartphone, especially at young age, constitutes a high-risk factor for many physical health problems. Several symptoms reported at follow-up were greatest among smartphone users; these include visual exhaustion, myalgia, neural brokenness, tension, visual and auditory inattentions [3-6]. Adolescents can be more vulnerable to the adverse effects of smartphone use because they are

Correspondence address: Marian M. Shafeek, Department of Physical Therapy for Pediatrics and Its Surgery, Faculty of Physical Therapy, Modern University for Technology and Information, El-Moustashar Mohammed Mostafa, El-Basatin Sharkeya, Qism El-Khalifa, Cairo, Egypt, e-mail: marian.magdy@pt.mti.edu.eg

Received: June 23, 2020

Accepted for publication: February 8, 2021

Citation: Shafeek MM, Battesha HHM, Wadee AN, Ibrahim HM. Influence of a smartphone use on dynamic balance in healthy adolescents. Hum Mov. 2022;23(2):76-83; doi: https://oi.org/10.5114/hm.2021.106171. 
uncritically receptive and easily adapted to new technologies, which can cause addictions similar to those of substances [7].

The balance study is essential for different ages, genders, races, and athletic abilities. Good posture, including static and dynamic balance, is very important for the adequate performance of many daily basic and recreational activities, so any balance alterations may lead to difficulties in the activates of daily living [8].

Different methods have been developed to assess posture stability, starting from the Timed Up and Go test, implemented by Mathias in 1986, which constitutes the shortest, simplest clinical balance test, though it is less objective [9]. After that, a forward-reaching test was used to assess the dynamic balance by measuring the maximum distance of reaching forward with either an arm or foot while remaining stable. That was followed by arm raising tests to measure a person's ability to maintain balance when raising and lowering the arms [10]. Another developed test to assess dynamic stability was the stepping test: the subject is asked to step a foot on and off a block as many times as possible in a detected time [10].

The methods of postural stability assessment should consider the effort needed to maintain the stability of dynamic balance. The total value of the stabilizing torque must counteract the destabilizing torque due to gravity in quiet standing [11]. The NeuroCom postural stability balance master systems have low to moderate reliability outcomes in measuring dynamic balance [12] but the assessment of postural stability with the Biodex Balance System constitutes the best selection for dynamic balance evaluation as it provides a valid, reliable, and repeatable objective assessment of balance on stable and unstable surfaces; it is also applied for training services. The device offers visual feedback of a patient's ability to control the body posture and enhance regaining the balance [13].

Another method to evaluate dynamic balance is the wobble board, which offers different biomechanical and neuromuscular control strategies that are more significant than balance tests on a firm surface. Differences in control strategies have implications for the understanding of various rehabilitation programs mechanisms [14]. In addition, balance performance measurement methods include static and dynamic balance tests in upright position standing which consider anthropometric characteristics, sex, and lower limb strength; these differently influence the Y Balance Test measures, regardless of limb dominance. The static and dynamic balances have been determined bilaterally by the Single Leg Stance Balance Test and the Star Excursion Balance Test, respectively. The latter provides scores for the anterior, posterolateral, and posteromedial directions, as well as an overall composite score [15].

Proper dynamic balance control is the basis in the achievement of motor skills. It mainly involves multiple strategies to minimize the displacements of the centre of gravity; these strategies are needed for numerous daily activities [16]. Many previous studies have discussed the effects of smartphone use on neck pain, cervical posture, muscle fatigue, gait, and many other aspects [17-19], but limited research has referred to the influence on dynamic balance and, to our knowledge, there are no studies concerning adolescents. Therefore, this study aimed to investigate the influence of smartphone use on dynamic balance in healthy adolescents. It was hypothesized that there was no influence (immediate or late) of smartphone use on dynamic balance in healthy adolescents.

\section{Material and methods}

\section{Participants}

A randomized controlled trial was conducted between August 2019 and October 2019 at the Laboratory of Biomechanics, Faculty of Physical Therapy, Modern University for Technology and Information, Cairo, Egypt. A total of 96 healthy adolescents of both genders (36 males and 60 females) participated in the study; they were recruited through online social media. The inclusion criteria involved age of 15-18 years, normal body mass index in accordance with the growth chart [20], and having been a smartphone user for at least 1 year. Excluded were all subjects who had a history of a disease affecting balance or neuromuscular control (cerebellum, basal ganglia, middle ear, proprioceptors), a musculoskeletal disorder, or even a complaint about any lower limb weakness. Each participant received a verbal explanation of all test procedures and applied them once before starting the proper test to become familiar with them. The subjects were randomly assigned, by coin tossing, to 2 groups (study and control group) (Figure 1).

\section{Procedures}

A Biodex system (Biodex Medical Systems Inc., Shirley, NY, USA, serial no: 13020193) was used for 


\section{HUMAN MOVEMENT}

M.M. Shafeek et al., Effect of smartphone on dynamic balance

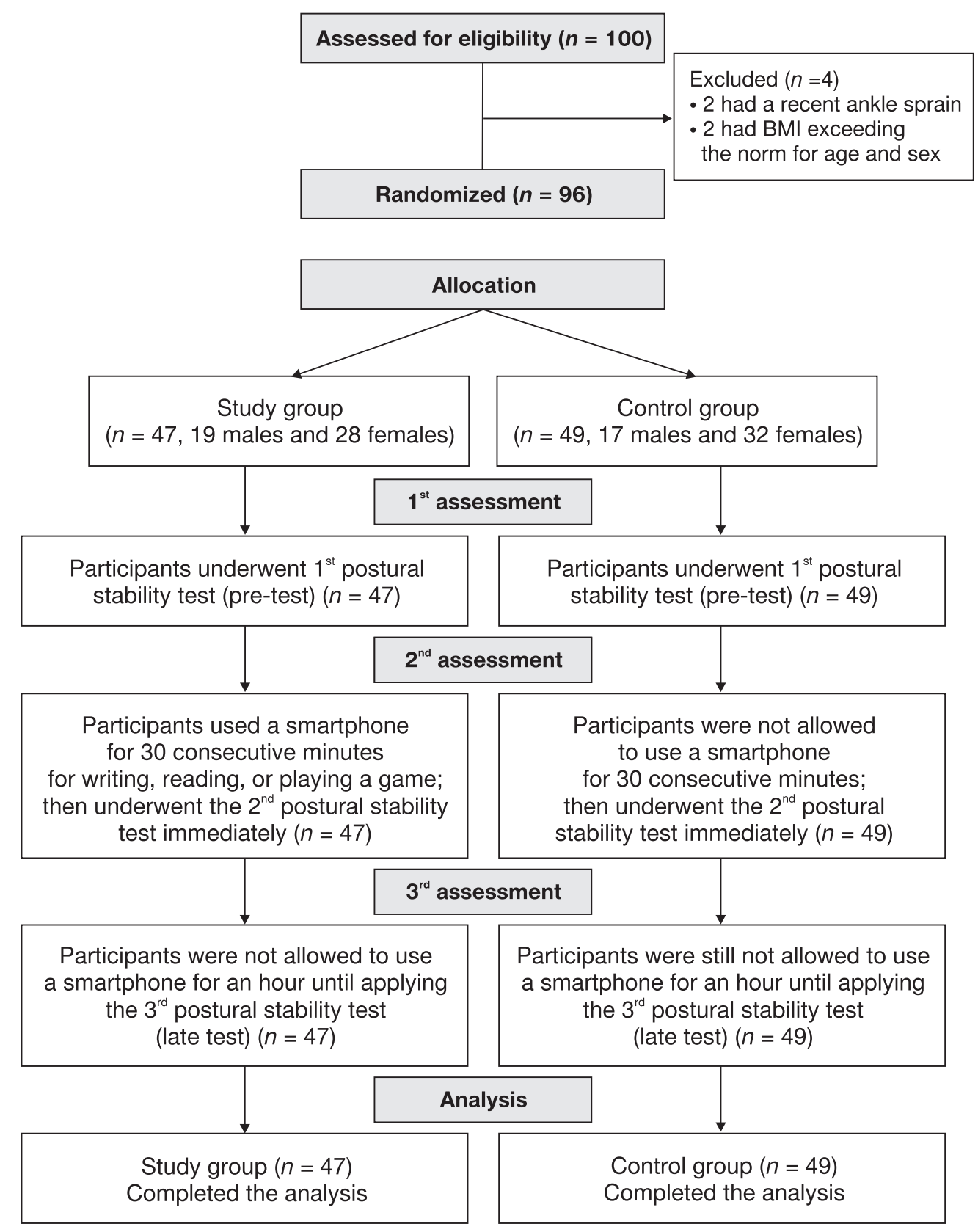

Figure 1 . The study flow chart

dynamic balance assessment. The components of Biodex include a circular foot platform with a diameter of 21.5 inches which permits $20^{\circ}$ tilting in all directions, a height-adjustable screen, height support rails, and a printer. The device allows static balance measurements plus 12 levels of dynamic balance measurements. Dynamic balance was assessed by the ability to control the tilting angle of the Biodex platform, which was reported as a stability index including the anteroposterior stability index (APSI), the mediolateral stability index (MLSI), and the overall stability index (OSI). Increasing values of the indices demonstrate a significant amount of sway, which implies a balance problem [21]. The Biodex system constitutes a valid and reliable objective measurement tool for dynamic balance assessment [22]. Regarding the smartphone, Galaxy Note 3 (SM-N900S, Samsung Electronics Co., Ltd., Seoul, Korea) was used (with Internet access connection).

The test procedures started by entering the participant's personal data (name, age, and height); then, the subject was asked to stand barefoot on the Biodex platform. After selecting the postural stability test, the level of stability was adjusted at the sixth level for 30 seconds (test period). When starting the test, the participant was asked to control their balance as much 
as possible with arms held at the sides, standing on both feet, with eyes open, and being guided by the visual feedback on the screen (the individuals were instructed to maintain the cursor in the centre of the circle displayed on the screen as much as possible). The measuring data included OSI, APSI, and MLSI. A trial was applied for familiarization with the test without recording its results; then, 3 recoded trials were conducted for each measurement, and the mean was obtained. A pre-intervention test was performed for all participants. In the study group, the individuals were allowed to use a smartphone for writing, reading, or playing a game for 30 consecutive minutes. Then, the immediate post-intervention test was performed. After that, the participants were not allowed to use a smartphone for an hour until conducting the late posture stability test. In the control group, the subjects were not allowed to use a smartphone for 30 minutes. Then the immediate post-intervention test was performed. After that, the participants were still not allowed to use a smartphone for an hour until conducting the late postural stability test (Figure 1).

\section{Statistical analysis}

All statistical calculations were carried out by using the IBM SPSS computer program, version 22 (IBM Corporation, USA). The sample size calculations were performed with the $G^{*}$ Power software (version 3.0.10). OSI was chosen as the primary outcome measure, while APSI was the secondary outcome. The effect size of OSI was estimated to be medium (0.25). The generated sample size of at least 40 participants per group would be required. Allowing for a $20 \%$ dropout rate, it was necessary to reach a total sample of a minimum of 96 participants. The test of homogeneity (Levene's test) showed that all data were homogenous. The test of normality (Shapiro-Wilk test) demonstrated that the data were normally distributed, so the parametric test was used (unpaired $t$-test to compare demographic data between groups and MANOVA to compare measurements within and between groups). The chi-squared test was applied for gender distribution, and the least significant difference test served for post-hoc comparison. The value of $p<0.05$ was considered statistically significant.

\section{Ethical approval}

The research related to human use has complied with all the relevant national regulations and institutional policies, has followed the tenets of the Declaration of Helsinki, and has been approved by the ethical

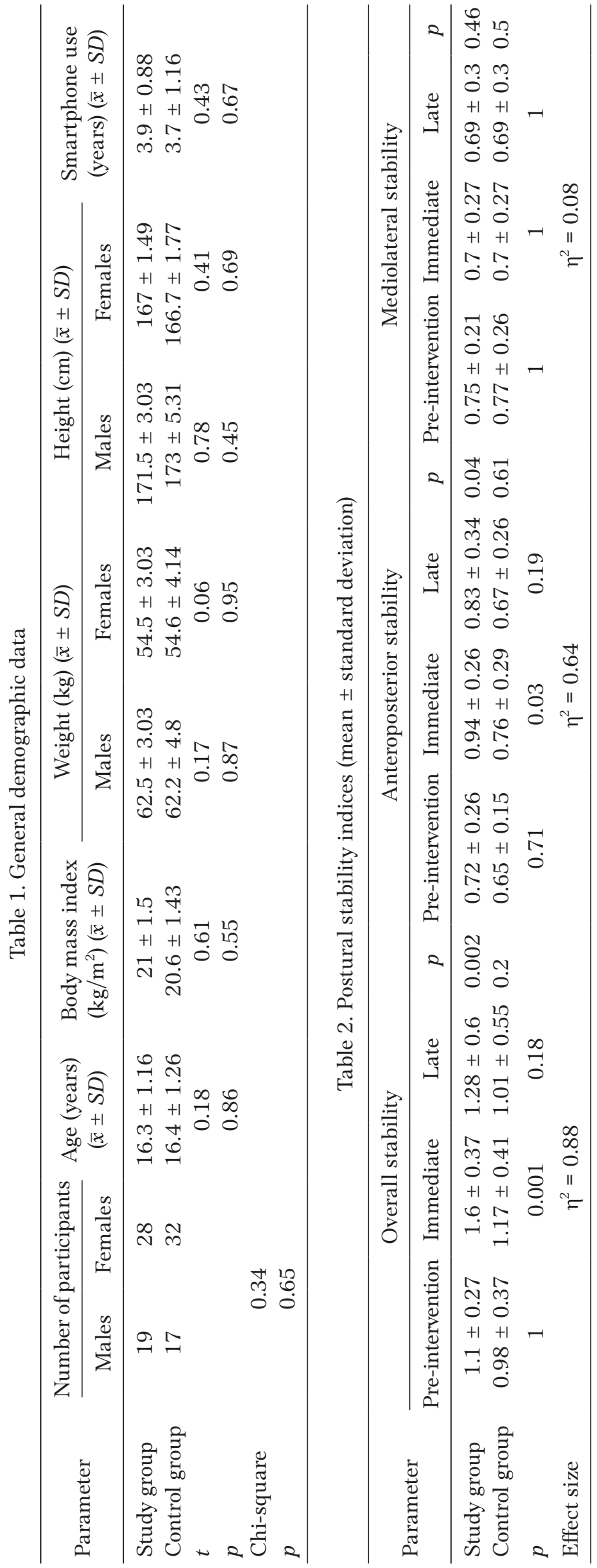


Table 3. Pairwise comparisons

\begin{tabular}{|c|c|c|c|c|c|c|}
\hline \multirow[t]{2}{*}{ Time X } & \multirow{2}{*}{ Time Y } & \multirow{2}{*}{$\begin{array}{l}\text { Mean difference } \\
\qquad(\mathrm{X}-\mathrm{Y})\end{array}$} & \multirow{2}{*}{$\begin{array}{l}\text { Slandered } \\
\text { error }\end{array}$} & \multirow{2}{*}{$p$} & \multicolumn{2}{|c|}{$\begin{array}{l}95 \% \text { confidence interval } \\
\text { for difference }\end{array}$} \\
\hline & & & & & Lower bound & Upper bound \\
\hline \multirow{2}{*}{ Pre-intervention } & Immediate & $0.37 *$ & 0.03 & $<0.001$ & 0.31 & 0.43 \\
\hline & Late & $0.36^{*}$ & 0.02 & $<0.001$ & 0.32 & 0.41 \\
\hline Immediate & Late & -0.012 & 0.02 & 0.560 & -0.06 & 0.03 \\
\hline
\end{tabular}

${ }^{*} p<0.05$

committee of the Faculty of Physical Therapy, Cairo University, Egypt (No. P.T.REC/012/002420). The study has been registered in the Pan African Clinical Trials Registry (No. PACTR201908659527420.

\section{Informed consent}

Informed consent has been obtained from all individuals included in this study and their legal guardians.

\section{Results}

\section{General demographic data}

The mean age, body mass index, weight (males and females), height (males and females), and duration of smartphone use ( \pm standard deviation) revealed that there were statistically insignificant differences between groups $(t=0.18,0.61,0.17,0.06,0.78,0.41$, and $0.43 ; p=0.86,0.55,0.87,0.95,0.45,0.69$, and 0.67 , respectively) (Table 1 ).

\section{Postural stability test}

There were significant effects of groups and measurements ( $p=0.0001, F=132.88 ; p=0.0001, F=68.39$, respectively, with hypothesis degree of freedom $=2$ ). An insignificant interaction was observed between groups and measurements ( $p=0.19, F=1.75$ ). The intra-group comparisons in the study group showed statistically significant differences in both OSI and APSI ( $p=0.002$ and 0.04 , respectively) and a statistically insignificant difference in MLSI $(p=0.46)$.

All postural stability indices (OSI, APSI, and MLSI) presented statistically insignificant differences $(p=0.2$, 0.61 , and 0.5 , respectively) within the control group.

The inter-group comparisons of both OSI and APSI revealed statistically insignificant differences in the pre-intervention and late measurements ( $p=1,0.18$, $0.71,0.19,1$, and 1 , respectively). There were significant differences in the immediate measurements of both OSI and APSI ( $p=0.0001$ and 0.03 , respectively) and a statistically insignificant difference in MLSI $(p=1)$. Partial eta squared was used to detect the effect size and it was found to be large for both OSI and APSI $\left(\eta^{2}=0.88\right.$ and 0.64 , respectively), while it was small for MLSI $\left(\eta^{2}=0.08\right)$ (Table 2$)$.

\section{Post-hoc least significant difference}

Pairwise comparisons showed statistically significant differences between the pre-intervention measurement and both the immediate and late measurements ( $p<0.001$ for both). There was a statistically insignificant difference between the immediate and late measurements $(p=0.56)$ (Table 3).

\section{Discussion}

The study showed statistically significant differences in both OSI and APSI ( $p=0.002$ and 0.04, respectively), as well as a statistically insignificant difference in MLSI ( $p=0.46$ ) within the study group. Statistically insignificant differences were observed in all postural stability indices (OSI, APSI, and MLSI; $p=0.2,0.61$, and 0.5 , respectively) within the control group. The study also revealed statistically insignificant differences in the pre-intervention and late measurements ( $p=1,0.18,0.71,0.19,1$, and 1 , respectively) for all postural stability indices, while there were statistically significant differences in the immediate measurements of both OSI and APSI ( $p=0.0001$ and 0.03 , respectively) and a statistically insignificant difference for MLSI $(p>0.05)$.

Regarding the significant decrease of OSI and APSI in the study group immediately after the smartphone use and the disappearance of this negative effect after 1 hour, these detected changes in the dynamic balance may be due to the influence of the smartphone on the information that flows through the interacting vestibular system, visual and proprioception information in the central nervous system [23]. Also, the electromagnetic waves of the smartphone result in 
a balance disturbance that occurs owing to the affection of visual and auditory factors. Because the afferent information required for normal body balance depends on superficial sensory perception and proprioception, any loss in these different body structures leads to increasing posture sway [19, 24].

The present study revealed statistically significant differences between the study and control groups in both OSI and APSI in the immediate measurements. These findings confirm that using the smartphone results in postural adjustments of neck and shoulder. This was discussed by Brown and Palvia [25], who concluded that neck pain and muscle tension after a smartphone use could change the sensitivity of neck proprioception as a result of muscle fatigue and increased loading of the neck and shoulder muscles due to the repeated motions of hands, wrists, and arms, all these factors affecting dynamic balance ability. The significant affection of the balance score in smartphone users may also be attributed to a disturbing cervical afferent function. Sustained muscle tension changes the sensitivity of neck proprioception, which affects the dynamic balance ability and increases posture sway [26]. A previous study found that cervical muscle fatigue caused a decrease in the dynamic balance owing to enhanced muscle spindle discharge, which occurred with muscle fatigue and obstructed the afferent feedback input to the central nervous system; this brought about changes in the proprioceptive and kinaesthetic properties of joints, which had a negative effect on the postural control ability [27].

Furthermore, Roy [28] showed that the major cause of balance alteration after an isometric contraction of cervical muscles appeared to be proprioceptive interference, which in turn increased the velocity of sway during quiet standing. Suboccipital muscle fatigue may change balance because of the activation of tonic gamma motor neurons due to the accumulation of metabolites during muscle contraction. The accumulation of potassium, as well as arachidonic and lactic acids leads to positive feedback, increased excitation of muscle spindles, and gamma motor system hyperactivity.

The study findings agreed with those presented by Cho et al. [29], who observed that using a smartphone could increase the instability of the dynamic postural balance. Therefore, smartphone use in such situations as walking or driving a vehicle should be discouraged. Also, Lamberg and Muratori [30] indicated that smartphone use had negative effects on gait pattern and parameters, as it decreased walking speed by $33 \%$ and increased lateral deviation during gait by $61 \%$ owing to reduced concentration.
Surprisingly, the current study revealed a significant immediate influence of smartphone use on OSI and APSI; there was also an insignificant influence on MLSI. This can be explained by the fact that balance is a complex motor control task involving the detection and integration of sensory information to assess the position and motion of the body in space and the execution of appropriate musculoskeletal responses to control body position within the context of the environment and task. Thus, balance control requires the interaction of the nervous and musculoskeletal systems and contextual effects [31, 32]. Although all the significant influences of the smartphone use on the dynamic balance appeared immediately, all disappeared after 1 hour, which implies that this impact is transient and improves through the interaction of body components, which helps to maintain balance by sensory detection of body motion (visual, vestibular, and somatosensory inputs), integration of sensorimotor information within the central nervous system, and execution of musculoskeletal responses $[19,33]$.

In contrast with the results found regarding mobile device use, researches examining the relation between physical activity, posture stability, and phone usage reported negative associations between mobile device use and physical activity: greater mobile device or application use was associated with declined physical activity and posture stability [34, 35]. Dual tasking while using different functions of a smartphone is widespread in the social life; it reduces the cognitive ability and thus affects postural control [36].

In addition, dynamic balance decreased in all 3 directions while playing games, sending messages, Web surfing, and listening to music using a smartphone. Playing games decreased cognitive ability most significantly, which resulted in the greatest decrease in dynamic balance. This was followed by sending a message, Web surfing, and listening to music [16].

Therefore, the hypothesis of immediate effect was rejected because the smartphone decreased the dynamic balance, while the hypothesis of late effect was accepted because the immediate decrease of dynamic balance was transient and the balance was regained an hour after smartphone use. Smartphone users should not perform activities that need a good balance immediately after using their smartphones for a long period (30 minutes). Also, trainers and sports educators should inform athletes to stop using their smartphones directly before sports activity as it could affect their dynamic balance during the participation in different sports. 


\section{Limitations}

Limiting the study sample size to only 96 subjects may affect generalization. The study is also limited to a specific age (adolescent) and to 30 minutes of smartphone activities, so more research is needed for different ages and periods of using a smartphone (shorter and longer than the investigated 30 minutes).

\section{Conclusions}

From the obtained results of this study, we conclude that dynamic balance could be decreased immediately after 30 consecutive minutes of using a smartphone for reading, writing, or playing games. Care should be thus taken to avoid any accidents while walking, sports participation, or other daily activities. This negative effect on dynamic balance can, however, disappear after 1 hour.

\section{Acknowledgements}

The authors thank Prof. Dr. Naguib Salem, Dean of Faculty of Physical Therapy, Modern University for Technology and Information, Cairo, Egypt, for allowing to carry out the study procedures at the Faculty Laboratory of Biomechanics.

\section{Disclosure statement}

No author has any financial interest or received any financial benefit from this research.

\section{Conflict of interest}

The authors state no conflict of interest.

\section{References}

1. Reda M, Rabie M, Mohsen N, Hassan A. Problematic Internet users and psychiatric morbidity in a sample of Egyptian adolescents. Psychology. 2012;3(8):626631; doi: 10.4236/psych.2012.38096.

2. Ma C, Li W, Cao J, Wang S, Wu L. A fatigue detect system based on activity recognition. In: Fortino G, Di Fatta G, Li W, Ochoa S, Cuzzocrea A, Pathan M (eds.), International Conference on Internet and Distributed Computing Systems. Cham: Springer; 2014; 303-311.

3. Park JS, Choi MJ, Ma JE, Moon JH, Moon HJ. Influence of cellular phone videos and games on dry eye syndrome in university students [in Korean]. J Korean Acad Community Health Nurs. 2014;25(1):12-23; doi: 10.12799/jkachn.2014.25.1.12.

4. Lee JH. Effects of forward head posture on static and dynamic balance control. J Phys Ther Sci. 2016;28(1): 274-277; doi: 10.1589/jpts.28.274.

5. Wilmer HH, Sherman LE, Chein JM. Smartphones and cognition: a review of research exploring the links between mobile technology habits and cognitive functioning. Front Psychol. 2017;8:605; doi: 10.3389/fpsyg.2017.00605.

6. Parasuraman S, Sam AT, Yee SWK, Chuon BLC, Ren LY. Smartphone usage and increased risk of mobile phone addiction: a concurrent study. Int J Pharm Investig. 2017;7(3):125-131; doi: 10.4103/jphi.JPHI_56_17.

7. Kim M-H, Min S, Ahn J-S, An C, Lee J. Association between high adolescent smartphone use and academic impairment, conflicts with family members or friends, and suicide attempts. PLoS One. 2019;14(7):e0219831; doi: 10.1371/journal.pone.0219831.

8. Howe TE, Rochester L, Neil F, Skelton DA, Ballinger C. Exercise for improving balance in older people. Cochrane Database Syst Rev. 2011;11:CD004963; doi: 10.1002/14651858.CD004963.pub3.

9. Yelnik A, Bonan I. Clinical tools for assessing balance disorders. Neurophysiol Clin. 2008;38(6):439-445; doi: 10.1016/j.neucli.2008.09.008.

10. Tyson SF, Connell LA. How to measure balance in clinical practice. A systematic review of the psychometrics and clinical utility of measures of balance activity for neurological conditions. Clin Rehabil. 2009;23(9): 824-840; doi: 10.1177/0269215509335018.

11. Chaudhry H, Findley T, Quigley KS, Bukiet B, Ji Z, Sims T, et al. Measures of postural stability. J Rehabil Res Dev. 2004;41(5):713-720; doi: 10.1682/jrrd.2003. 09.0140.

12. Pickerill ML, Harter RA. Validity and reliability of limits-of-stability testing: a comparison of 2 postural stability evaluation devices. J Athl Train. 2011;46(6): 600-606; doi: 10.4085/1062-6050-46.6.600.

13. Wilkerson GB, Behan E. Biodex integrated physical rehabilitation. Shirley: Biodex Medical Systems; 1994.

14. De Brito Silva P, Souza Oliveira A, Mrachacz-Kersting N, Laessoe U, Kersting UG. Strategies for equilibrium maintenance during single leg standing on a wobble board. Gait Posture. 2016;44:149-154; doi: 10.1016/j. gaitpost.2015.12.005.

15. Fusco A, Giancotti GF, Fuchs PX, Wagner H, da Silva RA, Cortis C. Y balance test: are we doing it right? J Sci Med Sport. 2020;23(2):194-199; doi: 10.1016/j. jsams.2019.09.016.

16. Hyong IH. The effects on dynamic balance of dualtasking using smartphone functions. J Phys Ther Sci. 2015;27(2):527-529; doi: 10.1589/jpts.27.527.

17. Lee S, Choi Y-H, Kim J. Effects of the cervical flexion angle during smartphone use on muscle fatigue and pain in the cervical erector spinae and upper trapezius in normal adults in their 20s. J Phys Ther Sci. 2017; 29(5):921-923; doi: 10.1589/jpts.29.921.

18. Samir SM, Elshinnawy AM, Abd Elrazik RK, Battesha HHM, El sayed Abdelkarem Ali M, Gazya AA. The long-term effect of smartphone overuse on cervical posture and range of motion in asymptomatic sedentary adults. J Adv Pharm Educ Res. 2019;9(4):89-95. 
19. Lee JH, Lee MH. The effects of smartphone multitasking on gait and dynamic balance. J Phys Ther Sci. 2018; 30(2):293-296; doi: 10.1589/jpts.30.293.

20. WHO Multicentre Growth Reference Study Group. WHO child growth standards based on length/height, weight and age. Acta Paediatr. 2006;450(Suppl.):76-85; doi: 10.1111/j.1651-2227.2006.tb02378.x.

21. Duecker JR. Measurement of validity for balance assessments using a modified CTSIB Sway Index versus a Biodex Sway Index. Master's thesis. Akron: University of Akron; 2013.

22. Drouin JM, Valovich-McLeod TC, Shultz SJ, Gansneder BM, Perrin DH. Reliability and validity of the Biodex System 3 Pro isokinetic dynamometer velocity, torque and position measurements. Eur J Appl Physiol. 2004; 91(1):22-29; doi: 10.1007/s00421-003-0933-0.

23. Ioannidou F, Hermens F, Hodgson TL. Mind your step: the effects of mobile phone use on gaze behavior in stair climbing. J Technol Behav Sci. 2017;2(3):109-120; doi: 10.1007/s41347-017-0022-6.

24. Cohen H, Blatchly CA, Gombash LL. A study of the clinical test of sensory interaction and balance. Phys Ther. 1993;73(6):346-351; doi: 10.1093/ptj/73.6.346.

25. Brown WS, Palvia P. Are mobile devices threatening your work-life balance? Int J Mob Commun. 2015; 13(3):317-338; doi: 10.1504/IJMC.2015.069128.

26. AlAbdulwahab SS, Kachanathu SJ, AlMotairi MS. Smartphone use addiction can cause neck disability. Musculoskeletal Care. 2017;15(1):10-12; doi: 10.1002/ msc. 1170 .

27. Alshahrani A, Aly SM, Abdrabo MS, Asiri FY. Impact of smartphone usage on cervical proprioception and balance in healthy adults. Biomed Res. 2018;29(12): 2547-2552; doi: 10.4066/biomedicalresearch.29-18594.

28. Roy G. Impact of mobile communication technology on the work life balance of working women - a review of discourses. J Contemp Manag Res. 2016;10(1):79-101.

29. Cho S-H, Choi M-H, Goo B-O. Effect of smart phone use on dynamic postural balance. J Phys Ther Sci. 2014; 26(7):1013-1015; doi: 10.1589/jpts.26.1013.

30. Lamberg EM, Muratori LM. Cell phones change the way we walk. Gait Posture. 2012;35(4):688-690; doi: 10.1016/j.gaitpost.2011.12.005.

31. Jelsma D, Ferguson GD, Smits-Engelsman BCM, Geuze RH. Short-term motor learning of dynamic balance control in children with probable developmental coordination disorder. Res Dev Disabil. 2015;38:213222; doi: 10.1016/j.ridd.2014.12.027.

32. Reyes A, Qin P, Brown CA. A standardized review of smartphone applications to promote balance for older adults. Disabil Rehabil. 2018;40(6):690-696; doi: 10.1080/09638288.2016.1250124.

33. Moral-Munoz JA, Esteban-Moreno B, Herrera-Viedma E, Cobo MJ, Pérez IJ. Smartphone applications to perform body balance assessment: a standardized re- view. J Med Syst. 2018;42(7):119; doi: 10.1007/s109 16-018-0970-1.

34. Leatherdale ST, Manske S, Faulkner G, Arbour K, Bredin C. A multi-level examination of school programs, policies and resources associated with physical activity among elementary school youth in the PLAY-ON study. Int J Behav Nutr Phys Act. 2010;7(1):6; doi: 10.1186/ 1479-5868-7-6.

35. Mojica CM, Parra-Medina D, Yin Z, Akopian D, Esparza L. Assessing media access and use among Latina adolescents to inform development of a physical activity promotion intervention incorporating text messaging. Health Promot Pract. 2014;15(4):548-555; doi: 10.1177/ 1524839913514441.

36. Shumway-Cook A, Woollacott M. Attentional demands and postural control: the effect of sensory context. J Gerontol A Biol Sci Med Sci. 2000;55(1):M10-M16; doi: 10.1093/gerona/55.1.m10. 